\title{
A NEW AIRBORNE LIDAR FOR REMOTE SENSING OF CANOPY FLUORESCENCE AND VERTICAL PROFILE
}

\author{
Ounis A. ${ }^{*}$, Bach J. ${ }^{1}$, Mahjoub A. ${ }^{2}$, Daumard F. ${ }^{3}$, Moya I. ${ }^{1}$, Goulas Y. ${ }^{1}$ \\ ${ }^{1}$ Laboratoire de Météorologie Dynamique, École Polytechnique, F91128 Palaiseau (France) \\ ${ }^{2}$ Laboratoire de Physique des Plasmas, École Polytechnique, F91128 Palaiseau (France) \\ ${ }^{3}$ INRA-EGC, Thiverval-Grignon (France) \\ *Email: ounis@lmd.polytechnique.fr
}

\begin{abstract}
We report the development of a new lidar system for airborne remote sensing of chlorophyll fluorescence $(\mathrm{ChlF})$ and vertical profile of canopies. By combining laserinduced fluorescence (LIF), sun-induced fluorescence (SIF) and canopy height distribution, the new instrument will allow the simultaneous assessment of gross primary production (GPP), photosynthesis efficiency and above ground carbon stocks. Technical issues of the lidar development are discussed and expected performances are presented.
\end{abstract}

\section{INTRODUCTION}

Terrestrial vegetation plays a fundamental role in the global carbon cycle; however, the coupling between climate change and plant eco-physiological functions still remains poorly understood. Experimental investigations at the leaf, canopy, ecosystem and regional scales are needed in order to improve climate predictions and mitigation strategies.

In this framework, current remote sensing techniques only deliver information on biophysical variables of the canopy, not on ecophysiological functions. ChlF is emitted by the photosynthetic pigments pool shortly after light absorption. It is considered as a promising remote observable of vegetation because of its close link with photosynthesis (for a review see [1]). Recently, a strong correlation was evidenced between SIF measured from space and GPP of various biomes derived from eddy covariance measurements at ground level [2]. However, GPP depends on both: (i) APAR, which itself depends on canopy structure, leaf biochemical composition and on incident radiation level, and (ii) the light use efficiency (LUE), which reflects the eco-physiological state of the plant [3]. On the other hand, SIF depends also on APAR and on the fluorescence quantum yield (FQY), which is linked to photosynthesis efficiency. Hence, disentangling APAR effects from LUE effects in GPP is possible if we are able to measure FQY independently. Assessment of relative FQY changes is feasible at canopy level by laserinduced fluorescence (LIF) if all measuring conditions are kept constant.

In this paper, we describe a new lidar concept for measurements of FQY and vertical profile (VP) of canopies (LASVEG, LASer VEGetation) combined to simultaneous SIF measurements from a single airborne platform. We discuss development issues and new possibilities that are open up by the future measurements.

\section{DERIVATION OF CANOPY PHOTOSYNTHESIS}

Our instrument aims to fill the gap between eddy fluxes towers and space observation:

- SIF gives access to GPP, a major component of carbon fluxes between surface and atmosphere [2] [4],

- LIF, as a proxy of FQY, allows assessment of fluorescence quenching, thus giving access to photosynthetic efficiency [5],

- lidar waveform maps above ground carbon stocks through the use of allometric relationships [6]. 


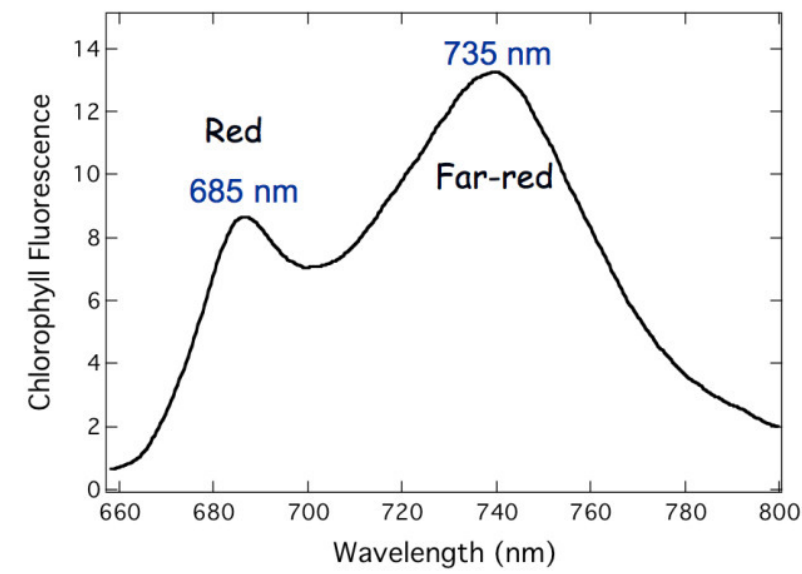

Figure 1: Chlorophyll fluorescence spectrum.

SIF and GPP share APAR as a common factor [4]. GPP is expressed as:

$$
G P P=A P A R \times L U E
$$

where LUE, as seen above, represents the fraction of absorbed PAR that is used for carbon fixation. On the other hand, SIF can be written as:

$$
S I F=A P A R \times F Q Y \times \tau_{F}
$$

where $\tau_{F}$ is the fraction of emitted fluorescence that escapes from the canopy. FQY can be assessed from LIF exitance considering Equation 3:

$$
L I F=I_{\text {laser }} \times f A P A R_{\text {laser }} \times F Q Y \times \tau_{\text {Flaser }}
$$

$I_{\text {laser }}$ being the laser intensity, $f A P A R_{\text {laser }}$ the fraction of laser light absorbed by vegetation and $\tau_{\text {Flaser }}$ the fraction of laser induced fluorescence that escapes from the canopy. As illumination geometry is constant in the case of LIF, these later factors are constant and LIF can be regarded as a proxy for FQY. In the far-red (see Figure 1), $\tau_{F}$ is close to one because fluorescence is not reabsorbed at these wavelengths. Hence, combining Equations 1 to 3 gives the possibility to investigate the relationship between LUE and FQY and to improve GPP modeling using fluorescence.

This instrument will also estimate the ground carbon stocks through the measurement of canopy vertical structure by lidar waveform analysis $[6,7]$. Biophysical variables such as
LAI, FAPAR and chlorophyll content will also be derived from the spectral reflectance [8].

\section{THE LIDAR SYSTEM}

\subsection{Signal to noise ratio}

Because of the low fluorescence yield $(<1 \%)$ of in vivo chlorophyll, the instrumental design needed to perform high-quality data become challenging. Therefore, we setup a ground test to investigate the overall detectability of the LIF. Using laser pulses of $9 \mathrm{~mJ} /$ pulse at $532 \mathrm{~nm}$ on a tree canopy situated at a distance of $42 \mathrm{~m}$, we achieved fluorescence measurements with $\mathrm{SNR}=100$. By increasing the laser pulse energy up to $30 \mathrm{~mJ}$, and the telescope diameter from $15 \mathrm{~cm}$ to $35 \mathrm{~cm}$, and by improving the detector response time $(+50 \%)$ and the stray light rejection $(+50 \%)$, a SNR of 100 at a flying altitude of $300 \mathrm{~m}$ should be achieved.

\subsection{The LASVEG excitation source}

The LASVEG system uses a compact and rugged lamp-pumped and frequency doubled Nd:YAG laser pulsed at a frequency of $20 \mathrm{~Hz}$ with $30 \mathrm{~mJ}$ energy per pulse (Ultra 50 from Quantel).

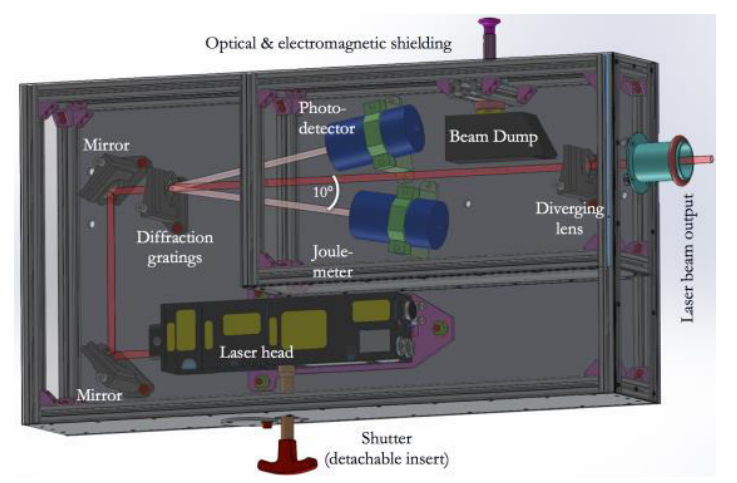

Figure 2: Schematic drawing of LASVEG excitation source.

Figure 2 shows a schematic drawing of LASVEG excitation source. The laser beam is expanded with a negative lens so that the illuminated area (foot print) is around $5-\mathrm{m}$ diameter for a typical operational flight-altitude of $300 \mathrm{~m}$. Such a beam-size is required to operate in eye-safety conditions on the ground level. Energy pulses are recorded with a pyroelectric joule-meter (ED 200, Gentec) in order to obtain normalized fluorescence and reflectance ratios. To record the energy of each pulse and trigger the data acquisition system we 
use the first order beams transmitted by a diffraction grating: at $532 \mathrm{~nm}$, these beams have a sampling ratio of $1.06 \%$ at an angle of $10^{\circ}$. A photo-detector is used to detect the presence of laser pulses.

\subsection{Eye safety experimental condition}

To avoid eye injury during operation, the energy received at eye level must be far under the Maximum Permissible Exposure. MPEs are defined by the international standard IEC 60825 , and depend on wavelength, pulses frequency and exposure time. This later parameter depends on spot size at ground level and aircraft speed. Figure 3 gives MPE as a function of illuminated spot diameter at ground level, compared to the exposure $\left(1.5 \mathrm{~mJ} \mathrm{~m}^{-2}\right)$ induced by the LASVEG source at $300 \mathrm{~m}$. It shows that eye-safety is achievable under good operational conditions.

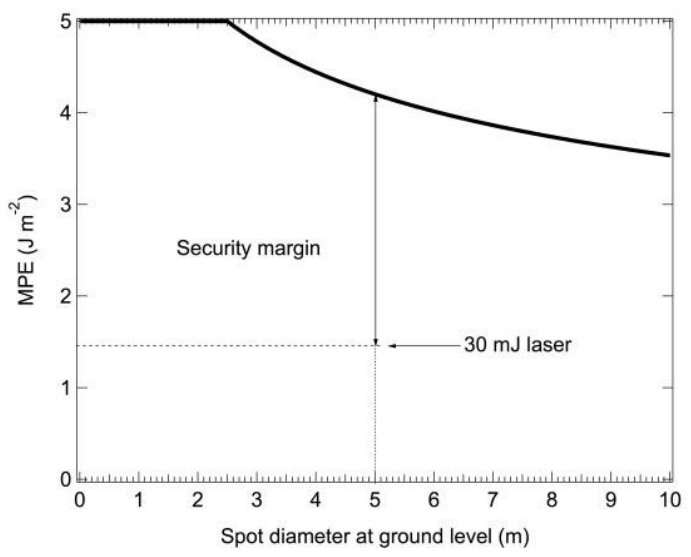

Figure 3 : Maximum Permissible Exposure as a function of illuminated spot size at ground level with aircraft speed at $50 \mathrm{~m} \mathrm{~s}^{-1}$.

\subsection{The LASVEG telescope}

Photon collection is performed with a Fresnel lens of $33 \mathrm{~cm}$ clear-aperture (Figure 4). Fresnel lenses have several benefits such as large aperture, large numerical aperture, reduced weight and low cost. In the other hand, commercial available telescopes are not suited for this kind of detection due to their typical numerical aperture. A set of 4 baffles is used to minimize stray-light. Fluorescence and back reflected-light are split into three channels by two dichroic mirrors: one channel at $532 \mathrm{~nm}$ for waveform analysis and two fluorescence channels at $685 \mathrm{~nm}$ and $740 \mathrm{~nm}$ (see Figures 4 \& 5).

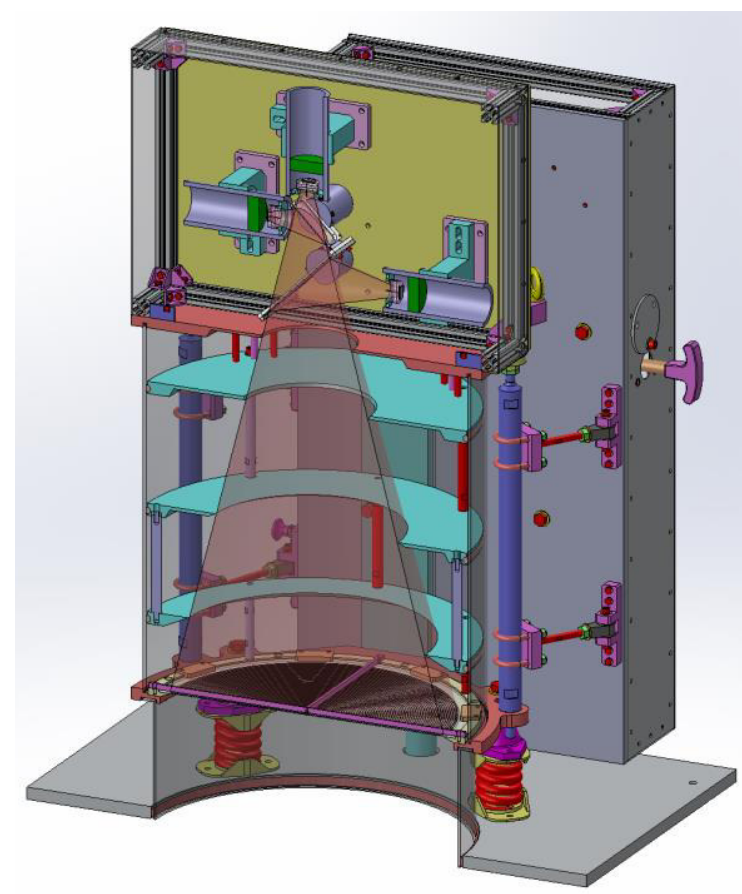

Figure 4: Schematic cross-section drawing of the LASVEG telescope.

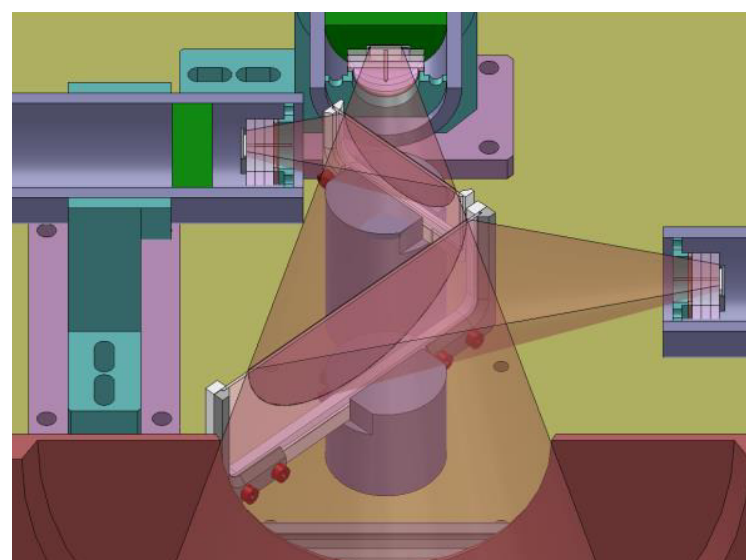

Figure 5: Schematic drawing of the LASVEG detector chamber with dichroïc mirrors.

The whole lidar system is mounted above the aircraft aperture through silent blocs.

\subsection{LASVEG detectors}

Three high-speed PIN photodiodes are used for fluorescence and waveform detection. Specific trans-impedance amplifiers have been developed to selectively amplify the pulsed signal and providing a high rejection of the background illumination $(\approx 50 \mathrm{~dB})$. The three signals are sampled at high rate $(250$ MSample/s) with a fast analog to digital card 
(NI 5761 coupled to a FPGA Module, National Instruments) and recorded on a hard disk (250 Go) for post processing. Peak values of fluorescence channels are divided by the pulse energy to give apparent fluorescence yield. The vertical structure of the canopy is deduced from waveform analysis of the reflected laser beam.

\section{PASSIVE INSTRUMENTS}

The SIF sensor is based on an improved version of the AIRFLEX sensor previously used in airborne campaigns [9]. AIRFLEX is a sixchannels high performance radiometer dedicated to measurements in the oxygen bands for fluorescence retrieval. Passive instruments include also a wide-band (400-1000 nm) spectrometer (from Ocean Optics) to record canopy reflectance. A synchronized video camera is used to record images of the scene that will help for target identification.

\section{CONCLUSIONS AND PERSPECTIVES}

We presented a new instrumental concept that combines measurements of LIF, SIF and vertical profile of canopies. This multiparameter instrument can simultaneously provide photosynthetic efficiency, gross instantaneous carbon flux and above ground carbon stock, which are major ecophysiological variables of canopy functioning. It is expected to resolve major issues in the improvement of GPP models based on fluorescence, with benefits for fluorescence space missions like GOSAT, OCO-2 or FLEX.

\section{ACKNOWLEDGMENTS}

This instrument is developed in the framework of the CALSIF project with the support of the French national agency ANR and the French space agency CNES.

\section{REFERENCES}

[1] G. Papageorgiou and Govindjee, Chlorophyll a fluorescence. A signature of photosynthesis. Dordrecht, The Netherlands: Springer, 2004.

[2] C. Frankenberg, J. B. Fisher, J. Worden, G. Badgley, S. S. Saatchi, J.-E. Lee, G. C. Toon, A. Butz, M. Jung, A. Kuze, and T. Yokota, "New global observations of the terrestrial carbon cycle from GOSAT: Patterns of plant fluorescence with gross primary productivity," Geophys. Res. Lett., 38(17), L17706, 2011.

[3] J. L. Monteith, "Solar radiation and productivity in tropical ecosystems," Journal of Applied Ecology, 9(3), 747-766, 1972.

[4] M. Rossini, M. Meroni, M. Migliavacca, G. Manca, S. Cogliati, L. Busetto, V. Picchi, A. Cescatti, G. Seufert, and R. Colombo, "High resolution field spectroscopy measurements for estimating gross ecosystem production in a rice field," Agricultural and Forest Meteorology, 150, 1283-1296, 2010.

[5] J. Flexas, J. M. Escalona, S. Evain, J. Gulias, I. Moya, C. B. Osmond, and H. Medrano, "Steady-state chlorophyll fluorescence (Fs) measurements as a tool to follow variations of net $\mathrm{CO} 2$ assimilation and stomatal conductance during water-stress in C-3 plants," Physiologia Plantarum, 114(2), 231240, Feb 2002.

[6] G. P. Asner, J. Mascaro, H. C. MullerLandau, G. Vieilledent, R. Vaudry, M. Rasamoelina, J. S. Hall, and M. van Breugel, "A universal airborne LiDAR approach for tropical forest carbon mapping," Oecologia, 168(4), 1147-1160, Apr 2012.

[7] J. Mascaro, M. Detto, G. P. Asner, and H. C. Muller-Landau, "Evaluating uncertainty in mapping forest carbon with airborne LiDAR," Remote Sensing of Environment, 115(12), 3770-3774, 2011.

[8] J. Verrelst, L. Alonso, G. Camps-Valls, J. Delegido, and J. Moreno, "Retrieval of Vegetation Biophysical Parameters Using Gaussian Process Techniques," IEEE Transactions on Geoscience and Remote Sensing, 50(5), 1832-1843, May 2012.

[9] I. Moya, F. Daumard, N. Moise, A. Ounis, and Y. Goulas, "First airborne multiwavelength passive chlorophyll fluorescence measurements over La Mancha (Spain) fields," in 2nd International Symposium on Recent Advances in Quantitative Remote Sensing: RAQRS'II, 25-29th September 2006, Torrent (Valencia)-Spain, 2006. 